\title{
Mucormycosis: An Uncommon Cutaneous Infection at Permanent Pacemaker-Implanted Site in a Very Low-Birthweight Baby
}

\author{
Minati Choudhury ${ }^{1}$ Jitin Narula ${ }^{1}$ Milind P. Hote ${ }^{1}$ Sarita Mohapatra ${ }^{1}$ \\ ${ }^{1}$ Department of Cardiac Anesthesiology and Critical Care, All India \\ Institute of Medical Sciences, New Delhi, India \\ J Card Crit Care 2021;5:242-245. \\ Address for correspondence Dr. Minati Choudhury, MD, PG Dip, \\ Department of Cardiac Anesthesiology and Critical Care, \\ Cardiothoracic Sciences Centre, All India Institute of Medical Sciences, \\ Ansari Nagar, New Delhi 110029, India \\ (e-mail: minati.2002@gmail.com).
}
Abstract
Keywords
- cardiac device infection
- mucormycosis
- pacemaker
- implantation

Permanent pacemaker implantation in low birthweight (LBW) babies with congenital complete heart block is extremely challenging due to a paucity of appropriate pulse generator placement pocket sites. The development of infection following an implantation procedure can pose a life-threatening risk to the patients. With more patients in the younger group receiving these devices than ever before and the rate of infection increasing rapidly, a closer look at the burden of infection and its impact on outcome of these patients is warranted. We report mucormycosis infection at the abdominal pacemaker pocket site of an infant requiring pacemaker explantation and re-insertion into the intrapleural space.

\section{Introduction}

Improvements in fetal imaging techniques have led to an increased number of indications for use of permanent pacemakers and implantable cardioverter-defibrillators in the newborns. This has been associated with an increased incidence of cardiac device infections, which could either be infection of the pulse-generator pocket or bloodstream secondary to systemic spread. ${ }^{1}$ Device infection remains a serious, life-threatening complication with rates varying between 0.5 and $5.1 \%$. Management of such avoidable complications is challenging especially in low birthweight (LBW) babies because of the paucity of ideal pacemaker pockets sites.

\section{Case Report}

A 32 days old neonate weighing 2,100 g with permanent pacemaker implantation done on day one of life for congeni- tal complete heart block ( - Fig. 1), presented with features of Staphylococcus aureus infection at the anterior abdominal wall-implanted pocket site on the eighth day of pacemaker placement. After a failed trial of intravenous antibiotics (cefotaxime and amikacin), the child was planned for pacemaker explantation and re-implantation in the left pleural cavity.

During the course of initial treatment, he developed left upper lobe pneumonia and severe uncompensated metabolic acidosis. On examination, there was fullness at pacemaker pocket site, soft tissue crepitus, and foul-smelling blackish crust. Transthoracic echocardiogram does not reveal any evidence of endocarditis. His blood sugar level was 290 $\mathrm{mg} / \mathrm{dL}$. In view of multiple air pockets, a possibility of an anaerobic infection was suspected along with the working diagnosis of mucormycosis with Staphylococcal pneumonia. Intraoperatively, abundant black foul-smelling crusts and necrotic tissue were found over the pacemaker implantation published online

January 14, 2022
DOI https://doi.org/

10.1055/s-0041-1739522. ISSN 2457-0206.

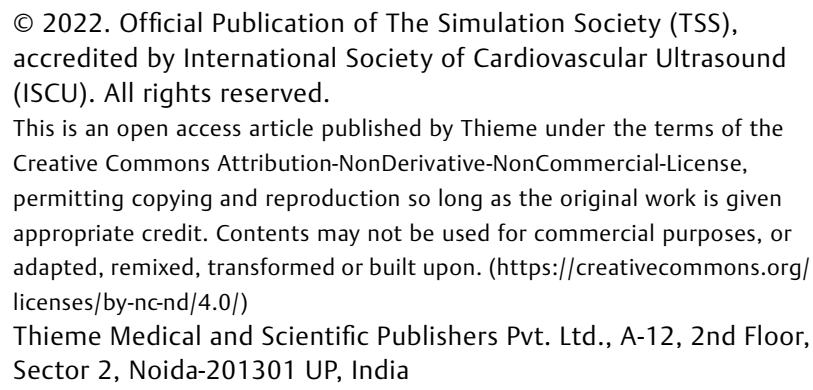

(C) 2022. Official Publication of The Simulation Society (TSS), accredited by International Society of Cardiovascular Ultrasound (ISCU). All rights reserved.

This is an open access article published by Thieme under the terms of the Creative Commons Attribution-NonDerivative-NonCommercial-License, permitting copying and reproduction so long as the original work is given appropriate credit. Contents may not be used for commercial purposes, or adapted, remixed, transformed or built upon. (https://creativecommons.org/ licenses/by-nc-nd/4.0/)

Thieme Medical and Scientific Publishers Pvt. Ltd., A-12, 2nd Floor, Sector 2, Noida-201301 UP, India 


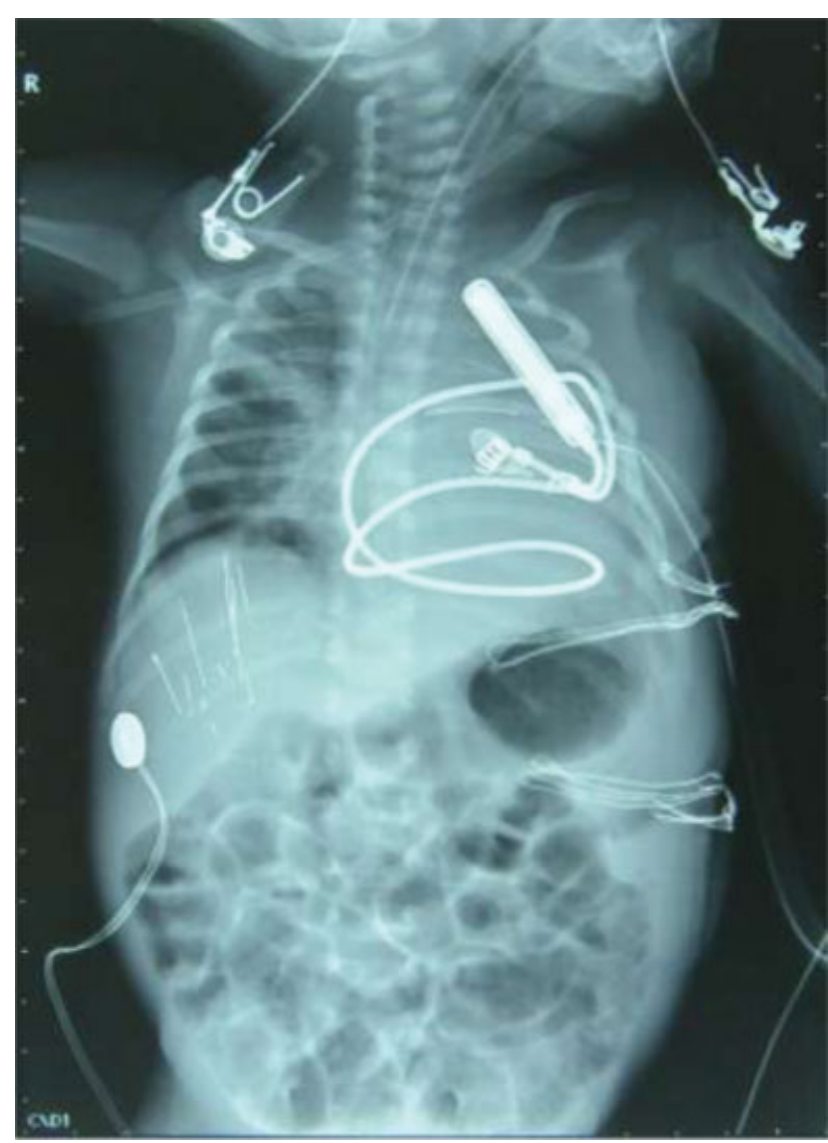

Fig. 1 Preoperative chest radiograph showing pacemaker placed in the anterior abdominal wall with a single epicardial pacing lead.

site. Potassium hydroxide preparation of the sample revealed thick aseptate fungal filaments strongly suggestive of mucormycosis. Aerobic culture yielded Escherichia coli sensitive to carbapenem and aminoglycosides. The pacemaker was explanted from the abdominal site and another pacemaker was implanted in the left pleural cavity (-Fig. 2). He was treated with intravenous amphotericin B infusion $(1 \mathrm{mg} / \mathrm{kg} /$ d) for 6 weeks. Antibiotic coverage was done with intravenous meropenem $20 \mathrm{mg} / \mathrm{kg} 8$ hourly for 7 days. He was weaned off from mechanical ventilation on third postoperative day and attained complete recovery after 8 weeks of monitoring and medication. Post hospital discharge, the child was not continued on any antifungal or antibiotics and was fine on his first follow-up.

\section{Discussion}

Cardiac device infection is a devastating complication associated with increased morbidity, mortality, and tremendous economic implications for hospital as well as patients. The local infection at the implanted pocket site usually present early, called pocket infection and usually managed conservatively with antibiotics and daily dressing. The second type is more serious type, associated with systemic infection usually present late and need call for a complete removal of all hardware, regardless of location. ${ }^{2}$ The largest series of pediatric pacemaker infection has been reported by Cohen

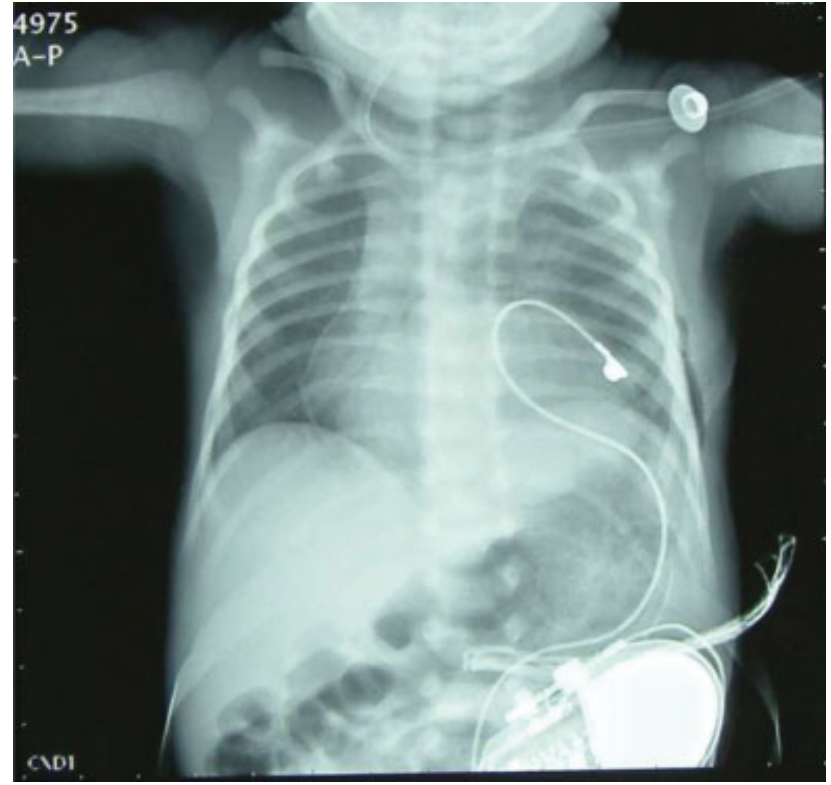

Fig. 2 Postoperative chest radiograph showing pacemaker device in left pleural cavity with a single epicardial pacing lead in situ.

et al. ${ }^{1}$ Staphylococcal species account for bulk of the infections in their series and all infections were bacterial. ${ }^{1}$ Most of them are superficial and children with Down's syndrome were found to be more prone for both superficial and deep infection.

Mucormycosis is a deep opportunistic mycosis caused by filamentous fungi, which is recognized as one of the most rapidly progressive lethal form of fungal infection in human beings with high mortality rate. This organism is mostly responsible for gas forming infection of osseous tissue and is associated with another bacterial infection, e.g., Salmonella, Staphylococcus, Streptococcus, and E. coli. ${ }^{3,4}$ Diabetic and immune compromised patients are more prone to this infection. Though our patient does not fall into this group, he had a high blood sugar level as well as pre-existing staphylococcal systemic infection.

Six distinct clinical varieties of mucormycosis can occur which can be classified as: rhino-orbito-cerebral, pulmonary, cutaneous, gastrointestinal, disseminated, and uncommon. The usual mode of infection is the result of inhalation of sporangiospores, inoculation of wounds, or ingestion. The primary site of infection varies according to the different genera included in the order of Mucorales and to the underlying disease of the patients. Pulmonary mucormycosis is more common in hematological malignancy patients, whereas rhino-orbito-cerebral type is associated with diabetes. Cerebral manifestation mostly happens in intravenous drug users. Cutaneous mucormycosis results from direct inoculation, mainly occurred after trauma or surgery, which happened in our case. In the cutaneous form, the disease is characterized by extensive necrotizing vasculitis, resulting in thrombosis, and subsequent tissue infarction. The typical presentation is induration of skin with surrounding erythema, rapidly progressing to necrosis giving rise to purulent discharge, 
and has moldy appearance. Once the infection sets in, subsequent angioinvasion by hyphae starts with a specific interaction with endothelial cells and systemic dissemination of the disease in $20 \%$ of the cases. Bacterial infection is often an association, which can delay the diagnosis of mucormycosis, which happened in our case too. .,6 $^{-6}$

Mucormycosis at a pacemaker-implanted site has not been reported before. The presence of air collections in the soft tissue could be due to infectious and noninfectious etiologies. It is predominantly associated with anaerobic pathogen or mixed infection. The diagnosis of mucormycosis almost always requires a histopathological evidence of fungal infection of the tissues. Obtaining a fungus in culture from a potentially infected site is rarely sufficient to establish the diagnosis of mucormycosis because the causative agent is ubiquitous, may colonize normal persons. Enzyme-linked immunosorbent assay, immunoblots, immune diffusion test, and molecular assays are used for diagnosis if histopathology and culture fail, and a suspicion of the infection is there from the clinical presentation.

Successful management of mucormycosis is based on a multimodal approach, including reversal or discontinuation of underlying predisposing factors if any, rapid correction of metabolic abnormalities, complete removal of all infected tissues, early administration of first-line antifungal agent (amphotericin B) at the optimal dose, and the use of various adjunctive therapies. Since our patient responded to the first line of treatment, it was continued during the course of management.

Corticosteroid and other immunosuppressant should be tapered quickly to the lowest possible dose if any. Posaconazole and isavuconazole are also active if patient cannot take amphotericin B due to some reason. Patients with existing renal dysfunction or premature renal system can be given liposomal amphotericin, which is less nephrotoxic than its contemporary. ${ }^{7}$ Itraconazole and terbinafine show some activity against certain strains. Though there is still not enough data to make a strong recommendation, the European Society of Clinical Microbiology and infectious disease/European Confederation of Medical Mycology guidelines recommend susceptibility testing to guide treatment of mucormycosis and to establish epidemiological knowledge. $^{8}$

Following removal of an infected device, the risk of infection reoccurrence is greater. So, it is important that patients are monitored closely. Serious watch for fever, redness, or drainage from the pocket site, or a drop in blood pressure should not be ignored.

In our case, the child was cachexic and intrapleural approach was used to avoid the anticipated difficulty in insertion with other techniques. Even in exceedingly small neonates, the chest cavity is large enough to accommodate the miniature pacemaker without difficulty. Some amount of lung collapse/consolidation that occur responds well to positive pressure ventilation. We implanted Microny II $\mathrm{SR}+$ (St Jude Medical Inc., Sylmar, California, United States) which weighed $12.8 \mathrm{~g}$ (volume $5.9 \mathrm{~mL}$ ). Single chamber ventricular pacing (VVI) mode was preferred over the dual chamber pacing, which requires placement of both atrial and ventricular leads and a larger generator device, which was technically difficult in such a small neonate. Stable lead impedance, sensing, and pacing thresholds were obtained at discharge.

\section{Conclusion}

This report highlights a rare case of cutaneous mucormycosis at the pacemaker implantation site of a neonate suffering from a primary staphylococcal infection at the local site followed by pneumonia. It most importantly emphasizes the need for an immediate intervention and management in such a case keeping in mind the possibility of a rapidly progressive fungal infection that might have systemic spread and fatal bony complication secondarily. The diagnosis and treatment of this infection remains a challenge. The clinical presentation at times is nonspecific, especially when superadded bacterial infection is present; and when it becomes apparent that the patient most probably has mucormycosis, it is often late to administer effective treatment. Early diagnosis is crucial and mostly based on histopathology and culture. Amphotericin B is the drug of choice and posaconazole also shows some promising results. Reversal of tissue hypoxia and immunotherapy may evolve in future leading to a better treatment of this devastating disease. Second, neonatal sepsis secondary to pacemaker device implant in LBW neonates leaves limited sites available for pulse generator placement. Small-sized devices have made intrapleural pacemaker insertion more feasible, safe, and easily applicable in patients with contraindication to abdominal/pectoral placement. The respiratory perturbations associated with the technique are usually well tolerated even in extremely LBW neonates.

\section{Conflict of Interest}

None.

\section{References}

1 Cohen MI, Bush DM, Gaynor JW, Vetter VL, Tanel RE, Rhodes LA. Pediatric pacemaker infections: twenty years of experience. J Thorac Cardiovasc Surg 2002;124(04):821-827

2 Baddour LM, Epstein AE, Erickson CC, et al; American Heart Association Rheumatic Fever, Endocarditis, and Kawasaki Disease Committee Council on Cardiovascular Disease in Young Council on Cardiovascular Surgery and Anesthesia Council on Cardiovascular Nursing Council on Clinical Cardiology Interdisciplinary Council on Quality of Care American Heart Association. Update on cardiovascular implantable electronic device infections and their management: a scientific statement from the American Heart Association. Circulation 2010;121(03):458-477

3 Jeong W, Keighley C, Wolfe R, et al. The epidemiology and clinical manifestations of mucormycosis: a systematic review and meta-analysis of case reports. Clin Microbiol Infect 2019;25 (01):26-34

4 Koklu E, Akcalcus M, Torum YA, Tulpar S. Primary gangrenous cutaneous mucormyosis of the scalp in a child. Pediatr Emerg Care 2008;24:242-243

5 Lelievre L, Gracia-Hermoso D, Abdoul H, et al. French mycosis study group. Medicine (Baltimore) 2014;93:395-404 
6 Francis JR, Villanueva P, Bryant P, Blyth CC. Mucormycosis in children: review and recommendation for management. J Pediatric Infect Dis Soc 2018;7(02):159-164

7 Perkhofer S, Lechner V, Lass-Flörl CEuropean Committee on Antimicrobial Susceptibility Testing. In vitro activity of isavuconazole against Aspergillus species and zygomycetes according to the methodology of the European Committee on Antimicrobial
Susceptibility Testing. Antimicrob Agents Chemother 2009;53 (04):1645-1647

8 Cornely OA, Arikan-Akdagli S, Dannaoui E, et al; European Society of Clinical Microbiology and Infectious Diseases Fungal Infection Study Group European Confederation of Medical Mycology. ESCMID and ECMM joint clinical guidelines for the diagnosis and management of mucormycosis 2013. Clin Microbiol Infect 2014;20(Suppl 3):5-26 